\title{
A compact configurable dual-band bandpass filter
}

\author{
Yu Guo ${ }^{1,4}$, Kexue Sun ${ }^{2}$, Xiaozhou Liu ${ }^{1 \mathrm{a})}$, Haodong $\mathbf{W} \mathbf{u}^{1 \mathrm{~b})}$, \\ Guojun Wang ${ }^{3}$, and Guann-Pyng $\mathbf{L i}^{4}$ \\ ${ }^{1}$ Key Laboratory of Modern Acoustics, Institute of Acoustics and School of Physics, \\ Nanjing University, \\ No. 22, Hankou Road, Nanjing, Jiangsu 210093, People's Republic of China \\ ${ }^{2}$ College of Electronic Science and Engineering, \\ Nanjing University of Posts and Telecommunications, \\ No. 66, Xinmofan Road, Nanjing, Jiangsu 210003, People's Republic of China \\ ${ }^{3}$ School of Management and Engineering, Nanjing University, \\ No. 22, Hankou Road, Nanjing, Jiangsu 210093, People's Republic of China \\ ${ }^{4}$ Department of Electrical Engineering and Computer Science, \\ University of California, Irvine, Irvine, CA 92697, USA \\ a)xzliu@nju.edu.cn \\ b)haodongwu@163.com
}

\begin{abstract}
A novel design of a configurable dual-band EBG (Electromagnetic Bandgap) bandpass filter is proposed. The EBG filter is manufactured by a conventional 2-layer PCB process in RO4350 substrate with surface mounted lumped capacitors. Specific working frequency of each band of the filter can be selected on demand by adjusting the value of corresponding capacitors, while the filter's performance and compact design is not compromised. The experimental results show a filter with size of $28.00 \times$ $28.00 \times 1.52 \mathrm{~mm}^{3}$ in $\mathrm{PCB}$ achieving performance of insertion loss of $2.18 \mathrm{~dB}, 2.24 \mathrm{~dB}$; return loss of $14.5 \mathrm{~dB}, 11.3 \mathrm{~dB}$ at $2.30 \mathrm{GHz}, 4.1 \mathrm{GHz}$ respectively. The filter has band to band isolation better than $48.0 \mathrm{~dB}$ and spurious free region up to $9.00 \mathrm{GHz}$.
\end{abstract}

Keywords: configurable, dual-band, EBG, bandpass filter

Classification: Microwave and millimeter wave devices, circuits, and systems

\section{References}

[1] X. B. Wei, Y. Shi, P. Wang, J. X. Liao, Z. Q. Xu and B. C. Yang: Electron. Lett. 48 (2012) 704. DOI:10.1049/el.2012.1107

[2] S.-R. Wu, K.-W. Hsu and W.-H. Tu: IET Microw. Antennas Propag. 6 (2012) 1422. DOI:10.1049/iet-map.2012.0257

[3] X. Z. Zhang, C. H. Chang, Q. Xue and B.-J. Hu: IEEE Microw. Wireless Compon. Lett. 20 (2010) 616. DOI:10.1109/LMWC.2010.2066553

[4] J. Xu, C. Miao, L. Cui, Y.-X. Ji and W. Wu: Electron. Lett. 48 (2012) 28. DOI:10.1049/el.2011.3081

[5] Y.-C. Chiou, C.-Y. Wu and J.-T. Kuo: IEEE Microw. Wireless Compon. Lett. 20 (2010) 67. DOI:10.1109/LMWC.2009.2038432 
[6] S. Luo, L. Zhu and S. Sun: IEEE Trans. Microw. Theory Techn. 58 (2010) 3427. DOI:10.1109/TMTT.2010.2053590

[7] Y. C. Li, H. Wong and Q. Xue: IEEE Microw. Wireless Compon. Lett. 21 (2011) 525. DOI:10.1109/LMWC.2011.2164394

[8] C.-Y. Chen and C.-Y. Hsu: IEEE Microw. Wireless Compon. Lett. 16 (2006) 246. DOI:10.1109/LMWC.2006.873584

[9] X. Y. Zhang and Q. Xue: IEEE Trans. Microw. Theory Techn. 55 (2007) 2183. DOI:10.1109/TMTT.2007.906501

[10] X. Gong, A. Margomenos, B. Liu, S. Hajela, L. P. B. Katehi and W. J. Chappell: IEEE Trans. Microw. Theory Techn. 52 (2004) 2557. DOI:10.1109/ TMTT.2004.837162

[11] W. J. Chappell, M. P. Little and L. P. B. Katehi: IEEE IMS 3 (2000) 1437. DOI:10.1109/MWSYM.2000.862244

[12] W. J. Chappell, X. Gong and L. P. B. Katehi: IEEE IMS 3 (2003) 1611. DOI:10.1109/MWSYM.2003.1210446

[13] W. J. Chappell, M. P. Little and L. P. B. Katehi: IEEE Microw. Wireless Compon. Lett. 11 (2001) 246. DOI:10.1109/7260.928927

[14] Y. Guo, S. Kim, X. Liu, H. Gao and G. P. Li: IEEE Trans. Compon. Packag. Manuf. Technol. 5 (2015) 668. DOI:10.1109/TCPMT.2015.2423294

\section{Introduction}

With an increasing demand for multipurpose wireless applications, more wireless systems are calling for multifunctional/multiband operations with support of a single broadband transceiver module. A multiband bandpass filter with compact size, planar configuration, and high performance is an integral part of a single transceiver implementation architecture.

The multiband filters have been researched intensively and their implementations can be classified into three main categories. The first category is to use two or more resonators with controllable fundamental and higher order resonant modes, such as stepped impedance resonators in dual-band BPFs [1], stub loaded SIR in dual-band BPF [2], stub loaded resonator in dual-band BPF [3], and quad mode resonators in quad band BPFs [4]. Generally, the nth resonant modes of each resonator need appropriate coupling for building up the nth passband. Since the resonant modes of such resonator are often dependent on each other, this method sometimes is difficult to place some resonant modes in desired or useful frequencies.

The dual-mode multiple band BPF has been an attractive candidate for multiple band applications owing to its compact size, simple physical layout, and design procedure. However, the dual-mode multiple band BPFs $[5,6,7]$ using a single resonator have reported a poor band to band isolation and notch like upper stopband of the second passband.

In the last category, multiple independently constructed single band filters working at specifically selected frequencies are combined to implement a multiple band filter by sharing input and output ports $[8,9]$. A good isolation between those bands has been achieved. However, the filters usually have an area multiple times larger than that of the single band filters. 
EBG filter/resonator $[10,11,12,13,14]$ has attracted a lot of attention in recent years due to its high Qu (Unloaded Quality Factor), ease of integration with other RF front end components and low cost. Most recently, a Compact Configurable EBG (CEBG) filter was demonstrated to function at specifically selected working frequency by simply adjusting an external capacitor by this group [14].

In this paper, a novel design of an EBG based dual-band filter is proposed to achieve targeted performance while alleviating the constraints imposed by aforementioned working frequency selection, band to band isolation, and size issues. To implement a compact dual-band EBG filter on demand, two single band filters with one set of external capacitors like the filter discussed in [14] are combined together. By sharing a common EBG substrate between the two, each filter has a better energy storage (i.e. higher $\mathrm{Qu}$ ) than it would have with a half size of the substrate. In addition, the use of two sets of external capacitors will not derail the design of EBG substrate for an individual filter. Specific working frequency of each band of the filter can be selected on demand by adjusting the value of corresponding capacitors, which determine the resonance frequency. Such a design of dual-band filter has size smaller than twice that of a single band filter and can work at multiple frequency bands without compromising individual band performance. In this paper, an implementation of a dual-band EBG filter with a standard two layer metal PCB technology is presented. Effectiveness of our design is verified by the agreement between simulation and measurement results.

\section{Design of the proposed dual-band filter}

Shown in Fig. 1(a) is 3D diagram of the proposed EBG filter to operate at dual bands and implemented in a common two-layer PCB. Energy transition and coupling circuit is designed on the top plate. It utilizes a Co-Planar Waveguide (CPW) to couple energy into the EBG substrate defined by the filter frame. For efficient coupling of energy to the EBG substrate, an electric short circuit at the end of the CPW and a co-existing electric open circuit of slotline are designed. Broadband energy transition and coupling is achieved by utilizing circular open slot as shown in Fig. 1(a), resulting in a relatively wide working frequency range in filter without performance degradation.

Electroplated hollow copper via holes serve as the periodic lattice in EBG substrate and connect the top layer with the bottom one. Air gaps separating metal pads from surrounding ground plane in the bottom copper layer are used for surface mounted capacitors as depicted in Fig. 1(b). A resonator is made up of the copper post, lumped capacitors shown in Fig. 1(b) and surrounding copper posts. Its equivalent circuit is shown in Fig. 1(c). By changing value of the lumped capacitors, working frequency of the corresponding resonator can be changed.

This proposed filter is combined by two single band filters made up of resonators. The first one consists of Res1 (Resonator1) and Res2 working at the same frequency and determines the first working frequency band of the dual-band filter, while Res3 and Res4 representing the second single band filter, and contributing to the other frequency band. Please note that these four resonators share a common EBG substrate without having interference between them. The 


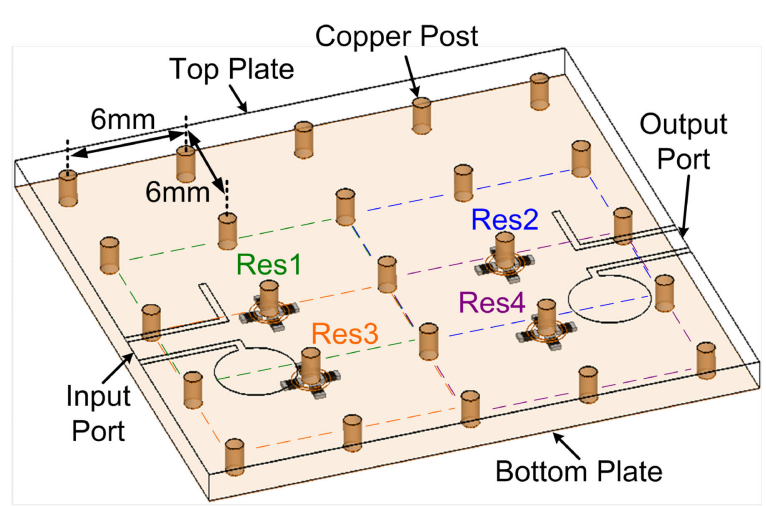

(a)

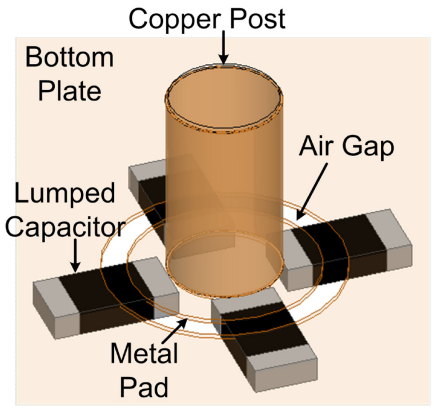

(b)

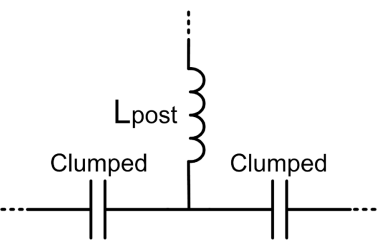

(c)

Fig. 1. Schematic of the proposed filter design. (a) 3D Diagram of proposed dual-band filter with generalized lumped capacitors attached on the bottom plate of the filter (b) Enlarged view of the attached lumped capacitors (c) Equivalent LC resonance circuit of Fig. 1(b).

ability to put two filters in close proximity to each other in this design leads to a compact size multiband filter.

Due to dependence of filter performance on the lumped capacitors' Q factor [14], a group of small value lumped capacitors is connected in parallel in each resonator for a relatively big capacitance value and high Q factor as shown in Fig. 1(b). Since the working frequency of the filter depends on the combined value of the capacitors, a relatively low operating frequency can be achieved in this compact filter design. Furthermore, an extremely wide spurious free region can be achieved in the EBG filter.

\section{Experimental results and discussions}

To demonstrate the proposed EBG filter, a full-wave simulation solver HFSS is used in theoretic design of dual-band bandpass filter operating at 2.3 and $4.1 \mathrm{GHz}$, and Rogers RO4350 substrate with a dielectric constant of 3.66, a thickness of $1.524 \mathrm{~mm}$ and loss tangent of 0.004 is used for experimental work. In implementing the filter, a standard two layer metal PCB process is used to construct the metal via posts, top and bottom metal plates.

Fig. 2 shows photographs of the fabricated filter with attached SMA connectors. ATC600L $0402(0.5 \mathrm{~mm} \times 1.0 \mathrm{~mm})$ series lumped capacitors were chosen for their small size and relatively high $Q$ factor in implementation of the proposed EBG 


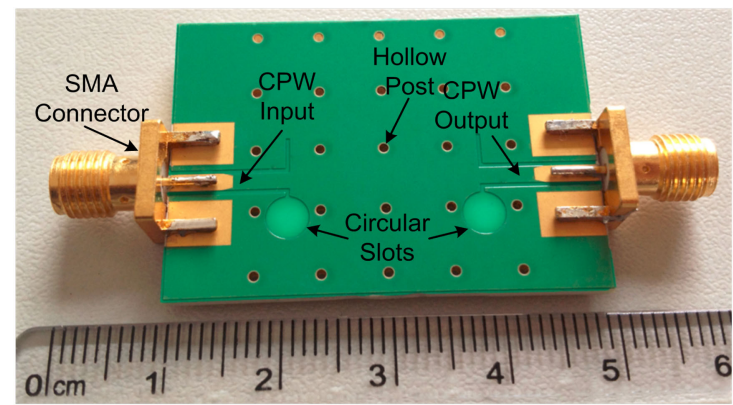

(a)

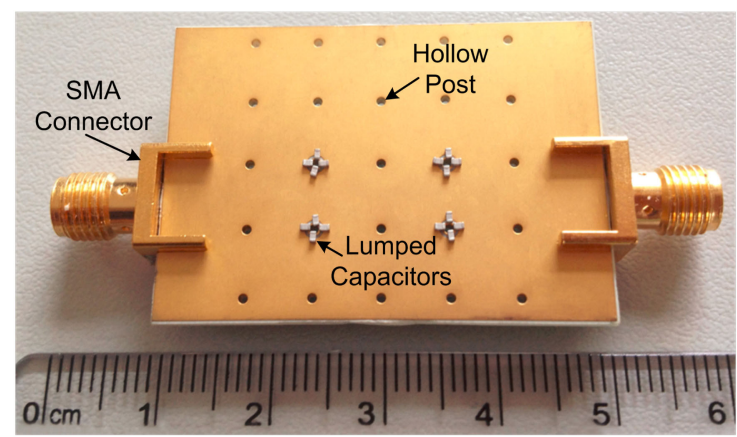

(b)

Fig. 2. Photographs of fabricated dual-band filter. (a) top view of filter (b) bottom view of filter.

bandpass filter. Bottom view of the filter loaded by ATC600L series capacitors is shown in Fig. 2(b). Working frequency of the bandpass filter can be easily adjusted by loading different value capacitors on the filter.

The fabricated filter was measured using an HP 8510C network analyzer. A short-open-load-through was adopted for calibration of measurements.

Insertion loss and $\mathrm{Qu}$ of the EBG filter depend on the $\mathrm{Q}$ factor of the surface mounted lumped capacitors which varies with their capacitance value and working frequency [14]. The simulated and measured results of the dual-band filter are shown in Fig. 3.

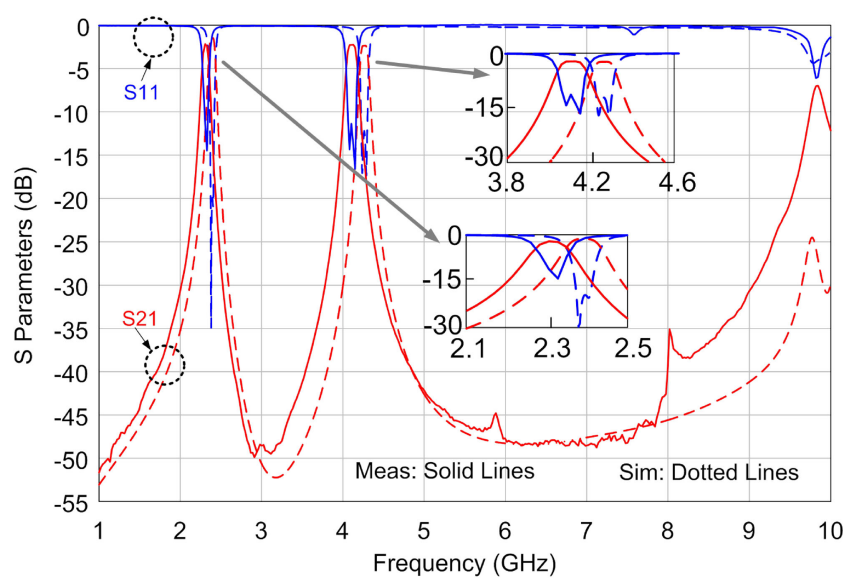


Excellent agreement was obtained where the filter exhibited two passbands. The tiny deviation between simulated and measured results may be attributed to the fabrication errors, the parasitic effects of the SMA connectors, and the parasitic capacitance or inductance introduced in manually soldering. The two passbands of the filter centered at 2.3 and $4.1 \mathrm{GHz}$ have $1 \mathrm{~dB}$ fractional bandwidths of $2.3 \%$ and $2.5 \%$, and measured minimum insertion losses of $2.18 \mathrm{~dB}$ and $2.24 \mathrm{~dB}$, respectively. Two passband return losses are better than $-14.0 \mathrm{~dB}$. The measured loss is dominated by the Q of lumped capacitors, which results in an effective Qu of 293 for the filter. From the measurement results, the band to band isolation of the filter is better than $48.0 \mathrm{~dB}$. The spurious signal suppression in the upper frequency band range from $4.5 \mathrm{GHz}$ to $9.0 \mathrm{GHz}$ is higher than $30 \mathrm{~dB}$.

\section{Conclusion}

In this letter, a novel configurable dual-band EBG filter with a compact size of $28.0 \times 28.0 \times 1.52 \mathrm{~mm}^{3}$ and operating at 2.3 and $4.1 \mathrm{GHz}$ was demonstrated in a standard PCB manufacturing technology. The measured results demonstrate good performance of the filter with respect to $\mathrm{Q}$ factor, insertion loss, return loss, band to band isolation and wide spurious free region. Working frequency of each band of the filter can be selected by simply changing the value of corresponding lumped capacitors. The current design can be extended to triple or quad bands filter with additional sets of resonators and a minute increase of total size of the filters, since they all share the same substrate. The current design of the filter also suggests that a compact high Q multiple band filter with tunable working frequency of each band be achievable, when the lumped capacitors are replaced by varactors or PIN diodes.

\section{Acknowledgments}

This work is supported by the national natural science foundation of China, (No. 11274166, No. 11474160). 